\title{
Effect of angiotensin/neprilysin inhibition on ventricular repolarization and clinical arrhythmogenesis
}

\author{
Gul S. ${ }^{1}$, Yontar O.C. ${ }^{1}$, Yenercag M. ${ }^{1}$, Seker O.O. ${ }^{1}$, Erdogan G. ${ }^{1}$, Arslan U. ${ }^{1}$ \\ ${ }^{1}$ University of Health Sciences Turkey, Samsun Training and Research Hospital, Cardiology Clinic, Samsun, Turkey
}

\begin{abstract}
Background: The most common reason for sudden cardiac death in heart failure is malign ventricular arrhythmias. LCZ 696 improves hospitalization and sudden cardiac death outcomes in heart failure, however mechanisms in preventing sudden cardiac death are still unknown. There is little information available assessing effect of LCZ 696 on Tp-e interval and related calculations. In this study, we aimed to investigate the impact of Sacubitril/valsartan therapy on Tp-e interval, Tp-e/QT ratio and Tp-e/QTc ratio in heart failure patients and its reflections on clinical arrhythmogenesis.

Methods: The study was designed as a prospective observational fashion. 265 patients with implantable cardioverter-defibrillator (ICD), who were on regular follow up at Samsun Training and Research Hospital Outpatient Heart Failure Clinic, were validated for the study. Clinical, echocardiographic, electrocardiogram and device data before initiation and six months after dose optimizing were obtained. Results: Stroke volume, cardiac output and ejection fraction significantly improved after LCZ 696 treatment. T wave related parameters, QT and QTC intervals significantly diminished. Furthermore, there was a negative correlation between baseline Tp-e interval duration and the absolute percentile increase of stroke volume(r:-0.234, p: 0.042) and cardiac output ( $r:-0.240, p: 0.037)$.

Conclusion: In our study, switching Renin-Angiotensin-Aldosterone-System inhibitor with Angiotensin receptor/neprilysin inhibitor was associated with increase in left ventricle performance and decrease of sustained ventricular arrhythmias that required ICD shocks. These positive findings were accompanied by improvements in surface electrocaridogram changes such as Tp-e and related indices.
\end{abstract}

Keywords: Tp-e, angiotensine/neprilysine inhibitor, arrhythmogenesis

Cite as Gul S., Yontar O.C., Yenercag M., Seker O.O., Erdogan G., Arslan U. Effect of angiotensin/neprilysin inhibition on ventricular repolarization and clinical arrhythmogenesis. Cardio-IT 2020; 7(1): e0103.

Received 10 May 2020. Accepted 30 May 2020.

(C) 2020, Gul S., Yontar O. C., Yenercag M., Seker O.O., Erdogan G., Arslan U.

Corresponding author: Osman Can Yontar. Samsun Eğitim ve Araştırma Hastanesi, Kardiyoloji Kliniği Barış Bulvarı Kadıköy Mahallesi No: 199 , PK: 55090

illkadım/SAMSUN/TURKEY Phone: +905056808747. Fax: +903622778765. E-mail: drcanyontar@gmail.com

\section{Introduction}

Sudden cardiac death is a major cause of death in reduced ejection fraction heart failure (HFREF) patients. The most common reason for sudden cardiac death in heart failure is malign ventricular arrhythmias [1]. PARADIGM-HF results exhibit that Sacubitril/valsartan decreases heart failure related hospitalizations and cardiovascular mortality by $20 \%$ in comparison to enalapil in patients with low ejection fraction, functional capacity 2-4 in New York Heart Association (NYHA) classification and is recommended as class 1 indication in heart failure treatment [2]. LCZ 696 improves hospitalization and sudden cardiac death outcomes in heart failure, however mechanisms in preventing sudden cardiac death are still unknown. PARADIGM-HF results demonstrate that Sacubitril/valsartan prevents sudden cardiac death by inhibiting the decrease in beneficial natriuretic peptides. In addition, study shows significant decrease in sustained and nonsustained ventricular arrhythmias, appropriate implantable cardioverterdefibrillator (ICD) shocks, frequency of premature ventricular beats, asystole and electromechanic dissociation. However, the precise mechanism by which LCZ696 causes a decrease in ventricular arrhythmias remains unclear.

$T$ wave peak to end (Tp-e) interval is new marker for ventricular vulnerability for arrhythmias and repolarization variability $[3,4]$. A prolonged Tp-e interval represents a period of potential vulnerability to re-entrant ventricular arrhythmias. It also has been associated with increased risk of mortality in the congenital and acquired long QT syndromes [5], in structural heart diseases such as hypertrophic cardiomyopathy [6] and mitral valve prolapse [7] and also in coronary heart disease patients such as undergoing primary percutaneous coronary interventions for myocardial infarction [8] and coronary ectasia [9].

There is little information available assessing effect of Sacubitril/valsartan on Tp-e interval and related calculations. In this study, we aimed to investigate the impact of Sacubitril/valsartan therapy on Tp-e interval, Tp-e/QT ratio and Tpe/QTc ratio in heart failure patients and its reflections on clinical arrhythmogenesis.

\section{Methods}

\section{Study participants}

Study was designed as a prospective observational fashion. Patients who admitted to Samsun Training and Research Hospital Outpatient Heart Failure Clinic and prescribed Sacubitril/valsartan were enrolled. Exclusion criteria were: absence of ICD, ventricular pacing percent $>40 \%$ or pacemaker dependent, presence of cardiac resynchronization therapy, left ventricle ejection fraction higher than 35\%, NYHA class lower than 2, left and/or right ventricle hypertrophy, left or right bundle branch block, QRS > 120 milliseconds on surface electrocardiogram (ECG), atrial fibrillation, ECG recordings that are insufficient for measurements, use of class I or III antiarrhythmics, having end stage renal of hepatic failure, not being on guideline directed optimal medical therapy for heart failure. Patients who were on guideline directed optimal medical therapy for heart failure were defined as:

1) use of maximum tolerated dose of renin-angiotensinaldosterone system (RAAS) inhibitor and beta blocker and ivabradine and mineralocorticoid receptor antagonist (MRA) for preceding at least six months before 
2) any therapeutical intervention was not expected for following at least six months

\section{Study Protocol}

From January 2017 to January 2020, 265 patients, who were on regular follow up at Samsun Training and Research Hospital Outpatient Heart Failure Clinic, were validated for study. After exclusions, remaining 76 patients were enrolled and analyzed (figure 1). As a general rule, all patients' RAAS inhibitors were stopped before Angiotensin Receptor/Neprilysin Inhibitor (ARNI) initiation and started with low dose (24/26 mg) for two weeks and then titrated to the maximum dose if tolerated. All patients' index (just before ARNI initiation) and follow up (at 9th month of ARNI initiation) ECGs and echocardiograms (ECHO) and ICD interrogation reports, blood chemistry and full blood count records, medications were obtained. The local institutional ethics committees reviewed and approved the study protocol. All procedures were carried out in accordance with the Declaration of Helsinki (2000).

\section{Electrocardiography}

A 12-lead surface ECG (Nihon Kohden Corporation, Cardiofax M Model ECG-1250, Tokyo, Japan) was performed in the supine position, with a $25 \mathrm{~mm} / \mathrm{s}$ paper speed and a voltage of 10 $\mathrm{mm} / \mathrm{s}$, at the beginning of the therapy and on the 9 th month. All of the ECG papers were scanned and transferred to the digital media, and the digital records were analyzed under $\times 400 \%$ magnification in a personal computer.

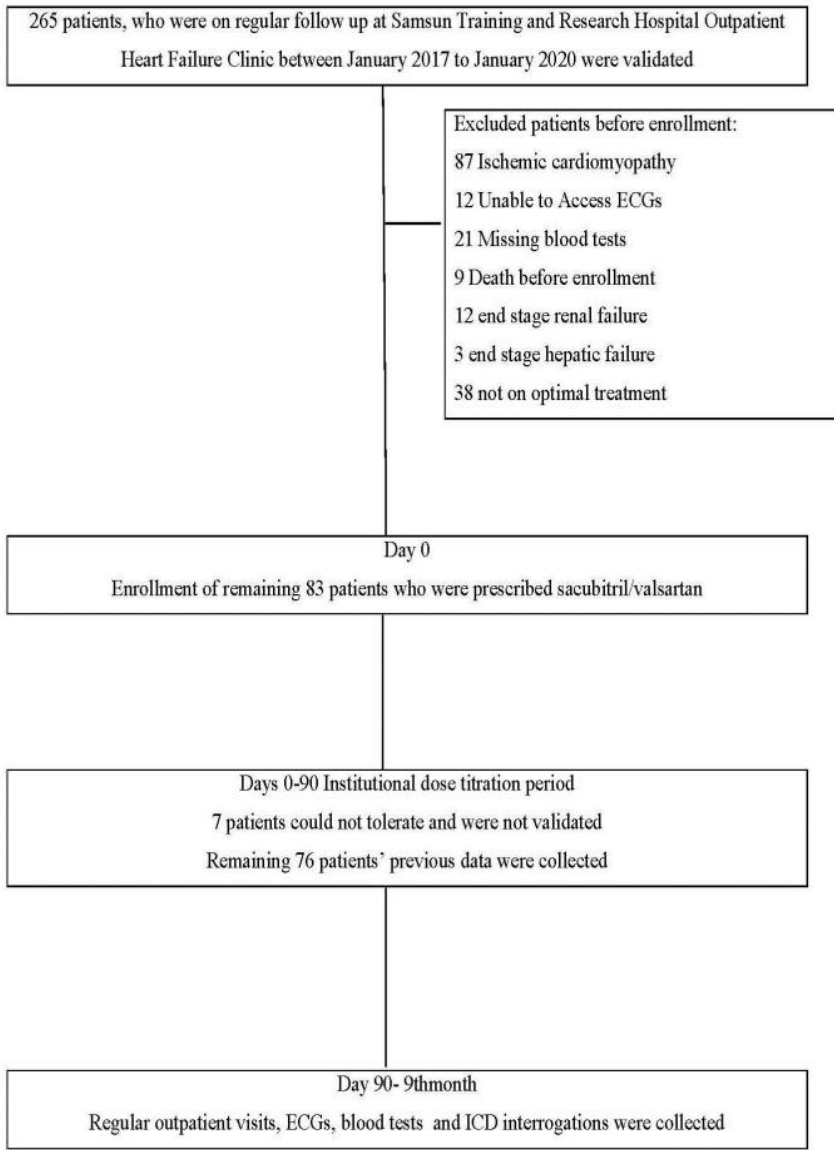

Figure 1. Flowchart of prospective observational study design
Patients having $\mathrm{U}$ wave and biphasic or negative $\mathrm{T}$ wave were excluded. Measurements were performed by two different cardiologists who were blinded to the patient data via using software after $\times 400 \%$ magnification. The QT interval was measured from the beginning of the QRS complex to the end of the T wave and also corrected QT (QTC) was calculated by using Bazett's formula (QTc = QT/ RR interval) [10]. Tp-e is measured from the peak of the $T$ wave to the end of the $T$ wave and it was performed from the precordial leads and an average value of at least two readings was calculated for each lead and measurement. QTc intervals were obtained using Bazett's formula (QTc = QT/RR-2). Then, Tp-e, Tp-e/QT, Tp-e/QTc were calculated.

All measurements were taken separately by two different cardiologists who were blind to the clinical and laboratory signs of the patients, and the mean values were transferred to database. The interobserver and intraobserver coefficients of variation were $3.2 \%$ and $2.4 \%$, respectively.

\section{Transthoracic Echocardiogram}

A complete transthoracic echocardiography study was performed using the GE Vivid E95 ultrasound system, with a frame rate of more than 60 frames/sec, before starting LCZ696 and at 9th month of therapy.

Left ventricular end-diastolic diameter (LVEDD), left ventricular end-systolic diameter (LVESD), interventricular septum (IVS) thicknesses and left atrial diameter (LAD) were measured from the parasternal long-axis view. Left ventricular ejection fraction (LVEF) was calculated by using the modified Simpson's method from the apical 4-chamber view. Stroke volume was calculated by using measured left ventricle outflow tract area and left ventricle outflow velocity. Estimated cardiac output was calculated by using following formula: Cardiac output $=$ (Stroke volume $\mathrm{x}$ resting heart rate)/1000. The operator was blinded to previous LVEF and clinical data.

\section{ICD interrogation data}

All patients' ICDs were interrogated with manufacturers' device routinely. Interrogation reports at least six months before and nine months after therapeutic intervention were obtained from routine device follow up. If there were any shock applied by device, number of shocks were noted and electrograms were printed and kept for further analysis.

\section{Statistical analysis}

Parametric data were expressed as mean (Standard deviation), and categorical data as percentages. SPSS 16.0 (SPSS, Inc., Chicago, Illinois, USA) was used to perform statistical procedures.

Baseline continuous clinical variables were expressed as percentages. Change in parametric data was compared using paired samples $T$ test. Temporal change of parametric data were evaluated by Wilcoxon signed rank test and nonparametric data of NYHA functional class was evaluated by chi square test. A $p$ value $\leq 0.05$ was accepted significant.

\section{Results}

Mean age for all participants was $66.8 \pm 8$. Maximum tolerated dose for ARNI was found to be 50 milligrams (mg) in 39 (51,3\%) patients and $100 \mathrm{mg}$ in $37(48,7 \%)$ patients (for all patients mean dose $74,3 \pm 25,1 \mathrm{mg}$, median dose $50 \mathrm{mg}$ ). Other baseline characteristics are demonstrated in table 1. 
Table 1. Baseline characteristics of patient group

\begin{tabular}{|l|c|}
\hline \multicolumn{1}{|c|}{ Parameter } & $\mathbf{n , ~ ( \% )}$ \\
\hline Male gender & $56(73.7)$ \\
\hline Diabetes & $35(46.1)$ \\
\hline Smoker & $39(51.3)$ \\
\hline Hypertension & $46(60.5)$ \\
\hline Hyperlipidemia & $47(61.8)$ \\
\hline Ischemic etiology for heart failure & $53(69.7)$ \\
\hline Mineralocorticoid antagonist usage & $47(61.8)$ \\
\hline Beta blocker usage & $72(94.7)$ \\
\hline Ivabradine usage & $39(51.3)$ \\
\hline Device brand: Medtronic & $40(52.6)$ \\
\hline Device brand: Boston Scientific & $19(25.0)$ \\
\hline Device brand: st. Jude Medical & $17(22.3)$ \\
\hline
\end{tabular}

Functional class distribution before ARNI was II-14 (18\%), III-55 (72\%) and IV- 7 (9\%) whereas it was II-30 (39\%), III-44 (57\%) and IV- $2(2 \%)$ with a significant improvement $(p<0.001)$. Temporal change of parameters such as ejection fraction, stroke volume, cardiac output and other relevant ECG parameters were listed in table 2. Stroke volume, cardiac output and ejection fraction were significantly improved after ARNI treatment. T wave related parameters and QT and QTC interval were significantly diminished (Table 2). Baseline Tp-e decrease from $90.9 \pm 5.0$ to $71.8 \pm 2.9$ $(p<0.001)$. Baseline Tp-e/QT and Tp-e/QTc ratios were also signicantly improved, from $0.20 \pm 0.01$ to $0.16 \pm 0.00$ and from $0.19 \pm 0.01$ to $0.15 \pm 0.00$, respectively ( $p<0.001$ for each). Furthermore, there was a negative correlation between baseline Tp-e interval duration and the absolute percentile increase of stroke volume(r:-0.234, p: 0.042) and cardiac output ( $r$ : -0.240 , p:0.037)

Device interrogation results also showed significant decrease in arrhythmias at ninth month after beginning of ARNI treatment. Before ARNI initiation, total amount of patients with arrhythmias were 32 (42.1\%). Twelve patients had ICD shocks for ventricular fibrillation, 9 patients had shocks for sustained ventricular tachycardia, and 11 patients had self terminating ventricular tachycardia without ICD intervention. During ARNI treatment period, $23(30.3 \%)$ patients had arrhythmia episodes which was consisted of 7 patient with ICD shocks for ventricular fibrillation, 5 patient with ICD shocks for ventricular tachycardia and 11 patients with self terminating ventricular tachycardia. Arhythmşa burden was significantly decreased with ARNI treatment (Table 2).

\section{Discussion}

Our findings demonstrated that Sacubitril/valsartan therapy, on top of guideline directed optimal medical treatment, improves Tp-e related indices on surface ECG. Another important finding is, there was a significant decrease in appropriate ICD shocks during the Sacubitril/valsartan treatment period. It is plausible to suggest that positive effect on repolarization may lead to an arrhythmic improvement.

Angiotensin receptor/neprilysin inhibitor (ARNI) modify effects of multiple endogenous vasoactive peptides, resulting in vasodilation, natriuresis, and inhibition of maladaptive ventricular remodelling [2]. Drugs that have potential to block the rapid component of the delayed rectifier potassium channel (IKR) coded by human Ether-à-go-go related gene (hERG) can prolong QTc, which may lead to increased susceptibility to cardiac arrhythmias, notably Torsades de Pointes [11-13].
Table 2. Echocardiographic and electrocardiographic parameters before and after sacubitril/valsartan initiation

\begin{tabular}{|l|r|r|c|}
\hline Parameters & \multicolumn{1}{l|}{ Before } & \multicolumn{1}{l|}{ After } & $\boldsymbol{p}$ value \\
\hline LVEF (\%) & $29.1 \pm 4.1$ & $30.5 \pm 4.6$ & 0.004 \\
\hline Stroke volume (ml) & $38.6 \pm 7.2$ & $41.9 \pm 6.3$ & $<0.001$ \\
\hline Cardiac output (L/min) & $2.6 \pm 0.4$ & $2.9 \pm 0.4$ & $<0.001$ \\
\hline Heart rate (bpm) & $69.5 \pm 6.4$ & $69.5 \pm 5.1$ & 0.951 \\
\hline ICD shocks (n) & $0.5 \pm 0.7$ & $0.3 \pm 0.4$ & 0.023 \\
\hline QT (msec) & $452.1 \pm 8.9$ & $449.7 \pm 8.7$ & $<0.001$ \\
\hline QTc (msec) & $470.0 \pm 0.2$ & $467.7 \pm 9.8$ & $<0.001$ \\
\hline Tp-e (msec) & $90.9 \pm 5.0$ & $71.8 \pm 2.9$ & $<0.001$ \\
\hline Tp-e/QT ratio & $0.20 \pm 0.01$ & $0.16 \pm 0.00$ & $<0.001$ \\
\hline Tp-e/QTc ratio & $0.19 \pm 0.01$ & $0.15 \pm 0.00$ & $<0.001$ \\
\hline
\end{tabular}

ICD: Implantable cardioverter defibrillator, LVEF; left ventricle ejection fraction, mm: millimeter, msec: millisecond, QTc; corrected QT, LVEDD: left ventricle end-diastolic diameter, LVESD; left ventricle end-systolic diameter, Tp-e: T wave peak to end interval. Data are presented as means \pm SD.

For this reason, drugs for heart failure are meticulously examined if they show a QT prolonging affect. Angiotensin II prolongs action potential duration probably by inhibiting fast activating components of potassium channels [14]

Nonetheless, clinical and experimental studies indicate that use of RAAS inhibitors have beneficial effects on QT interval [14] In some of in vitro studies regarding QT prolonging effects of sacubitril, potassium channel blocking action of the drug was not found to be associated with QT prolongation [15]. Furthermore, several studies on dogs and primates were unable to show a QT prolonging effect of sacubitril and metabolites [15]. Thomas et al. presented that even supratherapeutic doses of sacubitril (1200 $\mathrm{mg}$ ) did not differ from therapeutic doses $(400 \mathrm{mg})$ in terms of effect on QT interval [15]. Nonetheless, clinical and experimental studies indicate that use of RAAS inhibitors have beneficial effects on QT interval Similarly, our findings showed that after switching RAAS inhibitors with Sacubitril/valsartan combination, QT, and QTC intervals significantly got shortened (Table 2). This superior effect of ARNI in comparison to RAAS inhibitors affect may be a sum of many indirect factors, presumably may be a result of better left ventricle remodelling [16].

QT interval is a rather dynamic course that is the sum of ventricular depolarization and repolarization duration. Many factors such as electrolyte imbalance (potassium, magnesium, calcium), acidosis, several genetic diseases, drugs, and accompanying heart diseases contribute to changes in QT interval $[17,18]$. Recent studies indicated that Tp-e interval, which is the interval between the peak and the end of T wave on ECG can be used as an index of total (transmural, apico-basal, and global) dispersion of repolarization $[19,20]$ Also, increased Tp-e interval might be a useful index to predict ventricular tachyarrhythmia and cardiovascular mortality [21-23]. Recently, a new index, the Tpe/QT ratio has been suggested to be a more accurate measure for 
the dispersion of ventricular repolarization compared to QT, QTc and Tp-e intervals which is independent of alterations in heart rate [24]. Changes in these indices are frequent in many cardiac diseases and show increased susceptibility for ventricular arrhythmias $[25,26]$. Our main finding was significant decrease in Tp-e interval and Tp-e/QT, Tp-e/QTc after switching with sacubitril/valsartan combination (Table 2).

In their study Sen et al. [27] demonstrated that Tp-e interval length and ischemic etiology for heart failure were independent predictors of appropriate shocks in patients who underwent ICD implantations for primary protection. De Diego et al. demonstrated that Angiotensin-neprilysin inhibition decreased ventricular arrhythmias and appropriate ICDs shocks in heart failure with reduced ejection fraction patients under home monitoring as compared to angiotensin inhibition [28]. To our knowledge, the relationship between decrease in appropriate ICD shocks and novel repolarization indices Tp-e interval and Tp-e/QT ratio, are not studied in ARNI treatment before. We demonstrated that there was a significant decrease in appropriate ICD shocks when we compared preceding six months with next nine months after maximal tolerated ARNI initiation. Relatively small number of patients and also in our study prevented us to analyze if there was a relationship between Tp-e interval shortening and the decrease in appropriate ICD shocks. On the other hand, total number of ICD shock events were also very few for establishing a correlation.

In the presence of heart failure, enlarged myocardial tissue leads to elevated systolic wall stress. Consequently, left ventricle electrical heterogeneity occurs because of myocardial degeneration and fibrosis. Therefore, increased prevalence of ventricular arrhythmia is a reason of instability of heart muscle $[29,30]$. One may notice an increase in ICD shocks in patients with worsening heart failure and this is also an objective sign of poor prognosis. Although ICDs are cornerstone elements therapy for prevention of sudden cardiac death in heart failure, increase in appropriate or inappropriate shocks is related to increased mortality. Sacubitril/valsartan combination significantly decreases not only total mortality, but also sudden cardiac death in heart failure patients. Underlying mechanism for this effect is still unknown. However one can argue about the importance of drug's role in regression of fibrosis and remodeling process. One of the most favorable results of this effect is the improvement of cardiac output. Our results put forward that there was a significant increase in cardiac output after initiation of sacubitril/valsartan which is also significantly correlated with improvement in Tp-e and related repolarization parameters. Another important factor is, elevated natriuretic peptide levels are related with and independent predictors of sustained ventricular arrhythmia and ICD shocks. Although we do not have pre and post treatment serum natriuretic peptide levels, studies show a decrease of natriuretic peptides with sacubitril/valsartan combination [31]. One of other hypothetical mechanisms may be the decrease of myocardial wall stress by vasodilatation and diuresis. In addition, inhibition of RAAS leads to inhibition of symphatic system which plays major role in genesis and sustenance of malign ventricular arrhythmia.

Previous studies demonstrated the relevance of optimal medical therapy to clinical outcomes such as sudden cardiac death and ventricular arrhythmias [31]. In this observational study, sacubitril/valsartan was used according to international guidelines [32]. All patients were taking a RAAS inhibitor, which consequently was changed to ARNI. Among the patients, $100 \%$ took a beta blocker and $100 \%$ an MRA before and after ARNI. Of note, use of
MRA in our study was significantly higher than in other relevant trials such as PARADIGM-HF (55\%) [2] and DANISH (DANish randomized, controlled, multicenter study to assess the efficacy of Implantable cardioverter defibrillators in patients with nonischemic Systolic Heart failure on mortality) (58\%) [33]. Therefore, our population was previously well treated in terms of medical optimal therapy, which highlights the relevance of our observed ventricular arrhythmia reduction after the addition of sacubitril/valsartan. Additionally, after sacubitril/valsartan, patients had a significant improvement of NYHA functional class and left ventricular ejection fraction.

\section{Limitations}

Main limitation of our study is the prospective observational design. This forced authors to bound real life outpatient clinic routines and institutional rules which make difficult to obtain data in a wide spectrum. For example, this is the main cause of absence of pre and post treatment serum natriuretic peptide levels. Another limitation is the noninvasive measurement of cardiac output by echocardiography. Nonetheless, we think that is a safe and fast way to use echocardiography for determining contractility during patient follow up. Relatively short follow up period is one of remaining minor limitations. Also low number of participants is a limitation and brings out the need for larger trials to generalize our results.

\section{Conclusion}

In our study, switching RAAS inhibitor with ARNI was associated with increase in left ventricle performance and decrease of sustained ventricular arrhythmias and appropriate ICD shocks. These positive findings are also reflected by improvements in surface ECG changes such as Tp-e and related indices.

\section{Acknowledgement}

None.

\section{Statement of ethics}

Current study was approved by Samsun Training and Research Hospital Ethics Commitete.

\section{Disclosure statement None.}

\section{Funding Source}

Current study was not funded by any governmental or industrial agency.

\section{Author contribution}

SG: Study conception and design, OCY: Writing - Original Draft, Supervision MY: Data Curation, OOS: Validation, Methodology, GE: Formal analysis, Investigation

UA: Writing - Review \& Editing, Supervision 


\section{References}

1. Grabowski M, Ozierański K, Balsam P, et al. The effect of sacubitril / valsartan on the occurrence of ventricular arrhythmia and the risk of sudden cardiac death in patients with chronic heart failure with reduced left ventricular ejection fraction. Expert opinion of the Heart Rhythm and Heart Failure Sections of the Polish Cardiac Society. Kardiol Pol. 2019; 77(10): 987-993. https://pubmed.ncbi.nlm.nih.gov/31527563/

2. McMurray JJV, Packer M, Desai AS, et al. Angiotensine neprilysin inhibition versus enalapril in heart failure. N Engl J Med 2014; 371: 993-1004. https://pubmed.ncbi.nlm.nih.gov/25176015/

3. Opthof $\mathrm{T}$, Coronel R, Janse MJ. Is there a significant transmural gradient in repolarization time in the intact heart?: Repolarization Gradients in the Intact Heart. Circ Arrhythm Electrophysiol 2009; 2: 8996. https://pubmed.ncbi.nlm.nih.gov/19808447/

4. Antzelevitch $\mathrm{C}$, Sicouri S, Litovsky SH, et al. Heterogeneity within the ventricular wall. Electrophysiology and pharmacology of epicardial, endocardial, and M cells. Circ Res 1991; 69: 1427-1449. https://pubmed.ncbi.nlm.nih.gov/1659499/

5. Topilski I, Rogowski O, Rosso R, et al. The morphology of the QT interval predicts torsade de pointes during acquired bradyarrhythmias. $\begin{array}{llll}\text { J } A m & \text { Coll } & \text { Cardiol 2007; 49: }\end{array}$ https://pubmed.ncbi.nlm.nih.gov/17239713/

6. Shimizu $M$, Ino $H$, Okeie $K$, et al. T-peak to T-end interval may be a better predictor of high-risk patients with hypertrophic cardiomyopathy associated with a cardiac troponin I mutation than QT dispersion. Clin Cardiol 2002; 25: 335-339 https://pubmed.ncbi.nlm.nih.gov/12109867/

7. Yontar OC, Karaagac K, Tenekecioglu E, et al. Assessment of ventricular repolarization inhomogeneity in patients with mitral valve prolapse: value of T wave peak to end interval. Int J Clin Exp Med. 2014; 7(8): 2173-8 http://www.ncbi.nlm.nih.gov/pmc/articles/PMC4161563

8. Haarmark C, Hansen PR, Vedel-Larsen E, et al. The prognostic value of the Tpeak-Tend interval in patients undergoing primary percutaneous coronary intervention for ST-segment elevation myocardial infarction. J Electrocardiol 2009; $\quad$ 42: https://pubmed.ncbi.nlm.nih.gov/19643432/

9. Karaagac K, Yontar OC, Tenekecioglu E, et al. Evaluation of Tp-Te interval and Tp-Te/QTc ratio in patients with coronary artery ectasia. Int J Clin Exp Med. 2014; 7(9): 2865-70. https://doi.org/10.1093/cvr/cvu091.111

10. Van de Water A, Verheyen J, Xhonneux R, et al. An improved method to correct the QT interval of the electrocardiogram for changes in heart rate. J Pharmacol Methods. 1989; 22(3): 207-17.

11. Isbister GK, Page CB. Drug induced QT prolongation: the measurement and assessment of the QT interval in clinical practice. $\mathrm{Br} J \mathrm{Clin}$ Pharmacol 2013; 76(1):48-57. https://doi.org/10.1111/bcp.12040

12. Cubeddu LX. latrogenic QT abnormalities and fatal arrhythmias: mechanisms and clinical significance. Curr Cardiol Rev 2009; 5(3): 166176. https://doi.org/10.2174/157340309788970397

13. Redfern WS, Carlsson L, Davis AS, et al. Relationships between preclinical cardiac electrophysiology, clinical QT interval prolongation and torsade de pointes for a broad range of drugs: evidence for a provisional safety margin in drug development. Cardiovasc Res 2003; 58(1): 32-45

14. Wang $\mathrm{YH}$, Shi $\mathrm{CX}$, Dong $\mathrm{F}$, et al. Inhibition of the rapid component of the delayed rectifier potassium current in ventricular myocytes by angiotensin II via the AT1 receptor. Br J Pharmacol 2008; 154(2):429439. https://doi.org/10.1038/bjp.2008.95

15. Klimas J, Kruzliak P, Rabkin SW. Modulation of the QT interval duration in hypertension with antihypertensive treatment. Hypertens Res 2015; 38(7): 447-454. https://doi.org/10.1038/hr.2015.30

16. Langenickel $T H$, Jordaan $P$, Petruck J, et al. Single therapeutic and supratherapeutic doses of sacubitril/valsartan (LCZ696) do not affect cardiac repolarization. Eur J Clin Pharmacol. 2016; 72(8): 917-24. https://doi.org/10.1007/s00228-016-2062-9.
17. Mozos I. Laboratory markers of ventricular arrhythmia risk in renal failure. Biomed Res Int 2014; 2014: 509204. https://doi.org/10.1155/2014/509204

18. Singh JP, Johnston J, Sleight P, Bird R, Ryder K, Hart G. Left ventricular hypertrophy in hypertensive patients is associated with abnormal rate adaptation of QT interval. J Am Coll Cardiol 1997; 29(4): 778-84. https://doi.org/10.1016/S0735-1097(96)00576-1

19. Kors JA, Ritsema van Eck HJ, van Herpen G. Themeaning of the Tp-Te interval and its diagnostic value. J Electrocardiol 2008; 41:575-80. https://doi.org/10.1016/j. jelectrocard.2008.07.030

20. Antzelevitch C, Sicouri S, Di Diego JM, et al. Does Tpeak-tend provide an index of transmural dispersion of repolarization? Heart Rhythm 2007; 4: 1114-6. https://doi.org/10.1016/j.hrthm.2007.05.028.

21. Castro Hevia J, Antzelevitch C, Tornes Barzaga F, et al. Tpeak-tend and Tpeak-tend dispersion as risk factors for ventricular tachycardia/ventricular fibrillation in patients with the Brugada syndrome. J Am Coll Cardiol 2006; 47: 1828-34. https://doi.org/10.1016/j.jacc.2005.12. 049.

22. Smetana $P$, Schmidt $A$, Zabel $M$, et al. Assessment of repolarization heterogeneity for prediction of mortality in cardiovascular disease: peak to the end of the $T$ wave interval and nondipolar repolarization

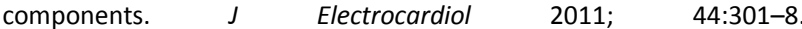
https://doi.org/10.1016/j.jelectrocard.2011.03.004.

23. Erikssen G, Liestol K, Gullestad L, et al. The terminal part of the QT interval ( $T$ peak to $T$ end): a predictor of mortality after acute myocardial infarction. Ann Noninvasive Electrocardiol 2012; 17:85-94. https://doi.org/10.1111/j.1542-474X.2012.00493.x.

24. Gupta P, Patel $C$, Patel $H$, et al. T(p-e)/QT ratio as an index of arrhythmogenesis. J Electrocardiol 2008; 41:567-74. https://doi.org/10.1016/j.jelectrocard.2008.07.016.

25. Waks JW, Tereshchenko LG, Parekh RS. Electrocardiographic predictors of mortality and sudden cardiac death in patients with end stage renal disease on hemodialysis. J Electrocardiol 2016; 49(6): 848-54. http://dx.doi.org/10.1016/j.jelectrocard.2016.07.020

26. Kalantzi K, Gouva C, Letsas KP, et al. The impact of hemodialysis on the dispersion of ventricular repolarization. Pacing Clin Electrophysiol 2013; 36(3): 322-7. http://dx.doi.org/10.1111/pace.12066

27. Sen O, Yilmaz S, Sen F, et al. T-peak to T-end Interval Predicts Appropriate Shocks in Patients with Heart Failure Undergoing Implantable Cardioverter Defibrillator Implantation for Primary Prophylaxis. Ann Noninvasive Electrocardiol 2016. https://doi.org/10.1111/anec.12383.

28. de Diego C, González-Torres L, Núñez JM, et al. Effects of angiotensinneprilysin inhibition compared to angiotensin inhibition on ventricular arrhythmias in reduced ejection fraction patients under continuous remote monitoring of implantable defibrillator devices. Heart Rhythm. 2018; 15(3): 395-402. https://pubmed.ncbi.nlm.nih.gov/29146274/

29. Jiménez-Candil J, Hernández J, Perdiguero $P$, et al. Prognostic Significance of Nonsustained Ventricular Tachycardia Episodes Occurring Early After Implantable Cardioverter-Defibrillator Implantation Among Patients With Left Ventricular Dysfunction. Am J Cardiol. $2016 \quad$ Nov 15; 118(10): 1503-1510. https://doi.org/10.1016/j.amjcard.2016.08.015.

30. Kubik M, Dąbrowska-Kugacka A, Lewicka E, et al. Predictors of poor outcome in patients with left ventricular noncompaction: Review of the literature. Adv Clin Exp Med. 2018 Mar;27(3):415-422. https://doi.org/10.17219/acem/67457

31. Bleyer AJ, Hartman J, Brannon PC, et al. Characteristics of sudden death in hemodialysis patients. Kidney Int 2006; 69: 2268-73. https://doi.org/10.1038/SJ.KI.5000446

32. Ponikowski P, Voors AA, Anker SD, et al. 2016. ESC Guidelines for the diagnosis and treatment of acute and chronic heart failure: The Task Force for the diagnosis and treatment of acute and chronic heart failure of the European Society of Cardiology (ESC). Developed with the special contribution of the Heart Failure Association (HFA) of the ESC. Eur J Heart Fail 2016; 18: 891-975. https://pubmed.ncbi.nlm.nih.gov/27206819/ 
33. Thune JJ, Pehrson S, Nielsen JC, et al. Rationale, design, and baseline characteristics of the DANish randomized, controlled, multicenter study to assess the efficacy of Implantable cardioverter defibrillators in patients with non-ischemic Systolic Heart failure on mortality (DANISH). Am Heart J. 2016; 179: 136-41. https://doi.org/10.1016/j.ahj.2016.06.016.

Authors:

Sefa Gul - University of Health Sciences Turkey, Samsun training and

Research Hospital, Cardiology Clinic, Samsun, TURKEY http://orcid.org/0000-0003-4809-7852

Osman Can Yontar - Associate Professor, University of Health Sciences Turkey, Samsun training and Research Hospital, Cardiology Clinic, Samsun, TURKEY http://orcid.org/0000-0002-0099-8654

Mustafa Yenercag - University of Health Sciences Turkey, Samsun training and Research Hospital, Cardiology Clinic, Samsun, TURKEY http://orcid.org/0000-0002-0933-7852

Onur Osman Seker - University of Health Sciences Turkey, Samsun training and Research Hospital, Cardiology Clinic, Samsun, TURKEY

Guney Erdogan - University of Health Sciences Turkey, Samsun training and Research Hospital, Cardiology Clinic, Samsun, TURKEY

Ugur Arslan - Associate Professor, University of Health Sciences Turkey, Samsun training and Research Hospital, Cardiology Clinic, Samsun, TURKEY 